\title{
Deficiency of Gankyrin in the small intestine is associated with augmented colitis accompanied by altered bacterial composition of intestinal microbiota
}

\author{
Toshiharu Sakurai $^{1^{*}+}$, Hiroki Nishiyama $^{2 \dagger}$, Tomoyuki Nagai $^{1}$, Susumu Goto ${ }^{3}$, Hiroyuki Ogata $^{2 *}$ and Masatoshi Kudo ${ }^{1}$
}

\begin{abstract}
Background: Gankyrin (GK) is an oncoprotein which regulates inflammatory responses and its inhibition is considered as a possible anti-inflammatory therapy for inflammatory bowel disease (IBD).

Methods: In this study, we investigated the role of GK in epithelial cells using mice with intestinal epithelial cellspecific GK deletion in (i) the entire small intestine and colon (Villin-Cre;Gankyrin f/f) and (ii) the distal intestine and colon (Cdx2-Cre;Gankyrin ${ }^{f / f}$ ).

Result: Unexpectedly, GK-deficiency in the upper small bowel augmented inflammatory activity compared with control mice when colitis was induced with dextran sodium sulfate. Biochemical analyses have revealed GKdeficiency to have caused reduction in the expression of antimicrobial peptides, a-Defensin-5 and -6, in the upper small bowel. Examination of human samples have further confirmed that the reduction of GK expression in the small bowel is associated with colonic involvement in human Crohn's disease. Through the sequencing of bacterial $16 \mathrm{~S}$ rRNA gene amplicons, bacteria potentially deleterious to intestinal homeostasis such as Helicobacter japonicum and Bilophila were found to be over-represented in colitis induced Villin-Cre;Gankyrin ${ }^{\text {fff }}$ mice when compared to Gankyrin $^{f / f}$ control mice under the same condition.
\end{abstract}

Conclusion: These results highlight the distinct site dependence of the pro- and anti-inflammatory functions of GK and provide important insights into the pathogenesis of IBD.

Keywords: Inflammatory bowel disease, Gankyrin, Microbiota, Helicobacter japonicum, Bilophila

\section{Background}

Inflammatory bowel disease (IBD) is characterized by a persistent inflammation in the colon and/or small intestine. Crohn's disease $(\mathrm{CD})$ may affect any segment of the digestive tract, while ulcerative colitis is restricted to the colon. IBD arises due to disruption of immune tolerance to the gut microbiota that leads to chronic intestinal inflammation and mucosal damage in genetically predisposed hosts $[1,2]$. The gut microbiota is known to be altered in patients with IBD and contributes to the

\footnotetext{
*Correspondence: sakurai@med.kindai.ac.jp; ogata@kuicr.kyoto-u.ac.jp ${ }^{\dagger}$ Toshiharu Sakurai and Hiroki Nishiyama contributed equally to this work.

${ }^{1}$ Department of Gastroenterology and Hepatology, Kindai University Faculty of Medicine, 377-2, Osaka-Sayama, Osaka 589-8511, Japan

${ }^{2}$ Institute for Chemical Research, Kyoto University, Uji 611-0011, Japan Full list of author information is available at the end of the article
}

pathogenesis of IBD. The incidence of IBD is increasing worldwide owing to the socioeconomic development and the environmental factors associated with modern life, that appear to promote the changes in gut microbiota $[1,2]$.

In IBD, long-standing inflammation is a major risk factor for colorectal cancer, also called as colitis-associated cancer (CAC) [3]. Indeed, patients with refractory IBD exhibit a higher risk of colorectal cancer than individuals in the general population [4]. In our previous reports, we found that chronic inflammation induces stress response proteins and Gankyrin (GK) (also known as PSMD10, p28 and Nas6p), which promote the development of CAC [5-8]. A significant correlation was found between the expression of these molecules and stem cell

(c) The Author(s). 2020 Open Access This article is distributed under the terms of the Creative Commons Attribution 4.0 International License (http://creativecommons.org/licenses/by/4.0/), which permits unrestricted use, distribution, and reproduction in any medium, provided you give appropriate credit to the original author(s) and the source, provide a link to the Creative Commons license, and indicate if changes were made. The Creative Commons Public Domain Dedication waiver (http://creativecommons.org/publicdomain/zero/1.0/) applies to the data made available in this article, unless otherwise stated. 
markers in the colonic mucosa of IBD patients. In addition, stress response protein heat shock protein A4 (HSPA4) and stem cell marker, B cell-specific Moloney murine leukemia virus insertion site 1 (Bmi1), could predict poor therapeutic response to steroid and anti-TNF$\alpha$ antibodies in IBD patients $[6,9]$. GK upregulates the expression of pro-inflammatory cytokines (TNF- $\alpha$ and IL-17) and stem cell markers, Bmil and sex-determining region Y (SRY)-box 9 (Sox9), in the colon. These data suggest that persistent inflammation can result in resistance to treatment and refractory clinical course in patients with IBD and eventually increases the risk of CAC through the major role played by GK.

To examine whether inhibition of GK might prevent colitis, we have employed 2 kinds of epithelial cellspecific GK-deficient mice, i.e. (i) small intestine and colon-specific GK-deficient mice (Villin-Cre;Gankyrin ${ }^{\text {f/f }}$ ) and (ii) distal intestine and colon-specific GK-deficient mice $\left(\mathrm{Cd} \times 2\right.$-Cre;Gankyrin $\left.{ }^{f / f}\right)$. These mice were challenged by dextran sodium sulfate (DSS) which is a colitis-inducing agent. Surprisingly, Villin-Cre;Gankyr$i^{f / f}$ mice were more susceptible to DSS-induced colitis and showed an alteration of gut microbiota in comparison with Gankyrinfff control mice. In contrast, $C d x 2$ Cre; Gankyrin $^{f / f}$ showed a similar level of inflammatory response as Gankyrin ${ }^{f / f}$ control mice. This study suggests that GK in the upper small bowel is involved in the pathogenesis of colitis through affecting gut microbiota.

\section{Methods}

\section{Knock out mice and treatment}

Gankyrin $^{f l o x} /$ flox $[8,10]$, Villin-Cre and Cdx2-Cre mice (obtained from Jackson laboratory) were used to produce tissue-specific conditional GK knockout mice, designated as Villin-Cre;Gankyrin ${ }^{f / f}$ and Cdx2-Cre;Gankyrin ${ }^{f / f}$ mice, respectively. All mice were maintained in a specific-pathogen-free environment and housed under a 12-h dark-light cycle (light from 7:00 to 19:00). They were given free access to standard diet and water and were not fasted before the experiments. Sex- and agematched Cdx2-Cre;Gankyrin ${ }^{f / f}$, Villin-Cre;Gankyrin ${ }^{f / f}$ [8], and Gankyrin ${ }^{f / f}$ (control) mice (8-16 weeks old) were administered with $2.5 \%(\mathrm{w} / \mathrm{v})$ dextran sodium sulfate (DSS; molecular weight, 36,000-50,000 kDa; MP Biomedicals, Solon, $\mathrm{OH}$ ) in drinking water for 7 days. Inflammatory cell infiltration score was assessed using a method described in a previous study (Villin-Cre;Gankyrin ${ }^{f / f}$ mice $n=6$, Gankyrin ${ }^{f / f}$ mice $n=5$ ) [5]. The pathological findings were blindly examined by TS and TN. Mice were sacrificed by cervical dislocation under deep isoflurane anesthesia. After the mice were sacrificed, the colon was excised from the ileocecal junction to the anus and the sections were longitudinally cut to prepare them for histological evaluation.
All animal procedures were performed according to approved protocols and in accordance with the recommendations for the proper care and use of laboratory animals. The animal study protocol was approved by the Medical Ethics Committee of Kindai University Faculty of Medicine and Institutional Animal Care (\#23-098, \#28-224).

\section{Human tissue samples}

The upper small intestine tissues $(n=20)$ were obtained by endoscopy from patients with $\mathrm{CD}$. Informed consent of all the patients was obtained. The clinical study protocol conformed to the ethical guidelines of the 1975 Declaration of Helsinki and was approved by the institutional review board of Kindai University Faculty of Medicine.

\section{Biochemical analyses}

Real-time qPCR and immunohistochemistry were performed as previously described in the respective references $[7,11,12]$. The primer sequences for mouse tumor necrosis factor (TNF)- $\alpha$, tumor growth factor (TGF)- $\beta$, Rspondin, wingless-type MMTV integration site 5B (Wnt5B), $\alpha$-Defensin 5 (Defa5), $\alpha$-Defensin 6 (Defa6), interferon (IFN) $-\alpha$, and IFN- $\gamma$ were: $5^{\prime}$-GACCAGGCTGTCGC TACATCA-3' and 5'-CGTAGGCGATTACAGTCACGG$3^{\prime} ; \quad 5^{\prime}$-GACTCTCCACCTGCAAGACC-3' and 5' GACTGGCGAGCCTTAGTTTG-3'; 5' CTGGAGAGGAACGA- $3^{\prime}$ and $5^{\prime}$-GTGCTCGATCTTGC ATTTGA-3'; 5'-CCGAGAGCGTGAGAAGAACT-3' , and 5'-GGCGACATCAGCCATCTTAT-3'; 5' -TATCTC CTTTGGAGGCCAAG-3' and 5'-TTTCTGCAGGTCCC AAAAAC-3'; 5'-GTCCAGGCTGATCCTATCCA-3' and 5'-GACACAGCCTGGTCCTCTTC-3'; 5' CTGACCCAGCAGAT-3' and 5'-GGTGGAGGTCATTG CAGAAT-3'; 5'-ACTGGCAAAAGGATGGTGAC-3' and 5'-GCTGATGGCCTGATTGTCTT-3'. The upper small bowel tissues from colitis non-induced Villin-Cre;Gankyrin ${ }^{\text {f/f }}$ mice and their corresponding floxed mice were collected and total RNA was extracted using RNeasy kit (QIAGEN, Hilden, Germany). Microarray analysis was performed by Hokkaido System Science Co., Ltd. (Sapporo, Japan).

\section{DNA extraction and sequencing}

Frozen samples of caecum and rectum were thawed and homogenized through the usage of Zirconia/Silica Beads (BioSpec Products) and MagNALyzer (Roche Diagnostics). Next, DNA was extracted from the homogenized samples by the usage of QIAamp DNA Mini Kit according to the manufacturer's instructions (Qiagen $\mathrm{GmbH}$, Hilden Germany). The variable V3-V4 16S rRNA gene regions of the extracted DNA samples were amplified by PCR with 16S Amplicon PCR Forward primer 5'TCGTCGGCAGCGTCAGATGTGTATAAGAGACAG 
-MID-GT-CCTACGGGNGGCWGCAG-3' and 16S Amplicon PCR Reverse primer 5'-GTCTCGTGGG CTCGGAGATGTGTATAAGAGACAG-MID-GT-

GACTACHVGGGTATCTAATCC-3'. The preparation of sequencing libraries was conducted according to the protocol described in '16S Metagenomic Sequencing Library Preparation: Preparing 16S Ribosomal RNA Gene Amplicons for the Illumina MiSeq System' protocol [13] with the usage of the Nextera XT Index Kit (Illumina). The MiSeq Reagent Kit v2 (300 cycles) and MiSeq (Illumina, San Diego, CA, USA) device was used for the sequencing of the samples.

\section{Bioinformatics analysis of $16 \mathrm{~S}$ rRNA amplicon sequences}

Amplicon sequences were processed with the following procedures modified from our previous paper [14]. Lowquality and primer regions were removed from each paired-end reads using Trimmomatic (version 0.35) (PE, SLIDINGWINDOW:40:15, MINLEN:50) [15] and Cutadapt (version 1.11) (-e 0.18, --pair-filter = both) [16], respectively. Paired-end reads were aligned to make merged reads (i.e. "reads" hereafter) using FLASH (version 1.2.11) (-m 30, -M 271, -x 0.25) [17].

The representative 16S rRNA sequences of Greengenes database (version 13_8, 99_otus.fasta) [18] were used to cluster reads into OTUs at $99 \%$ identity using UCLUST [19] implemented in Quantitative Insights Into Microbial Ecology's (QIIME version 1.9.1) [20] parallel_pick_otus_ uclust_ref.py (--max_rejects 0 , --enable_rev_strand_ match). Taxonomic groups were assigned to OTUs based on '99_otu_taxonomy.txt' in Greengenes database. Reads that were not assigned to OTUs at this stage were checked for chimera using uchime algorithms [21] implemented in VSEARCH (version 2.10.4), (--uchime_denovo and --uchime_ref, --db 99_otus.fasta) [22]. Identified chimeric sequences were discarded. Remaining unclassified reads were grouped into additional OTUs at 99\% identity using USEARCH [19] implemented in QIIME's pick_otus.py (--otu_picking_method usearch61, --similarity 0.99, --enable_rev_strand_match). Of these OTUs, singletons and doubletons were discarded. Taxonomy was assigned to the most abundant sequence of each OTU using the RDP classifier [23] in QIIME's parallel_assign_taxonomy_ rdp.py (--confidence 0.8, --id_to_taxonomy_fp 99_otu_ taxonomy.txt, --reference_seqs_fp 99_otus.fasta) with training on Greengenes database.

Rarefaction curves were generated by randomly sampling reads from each sample 10 times and calculating the average number of OTUs across different sampling depths with intervals of 5000 reads using QIIME's parallel_multiple_rarefactions.py. OTU richness and Shannon diversity index [24] values were calculated based on reads randomly sampled at the depth of the smallest sample size with QIIME's alpha_rarefaction.py. This was repeated 10 times for each sample and their average was used for the non-parametric two-sample $t$-test implemented in QIIME's compare_alpha_diversity.py with 10, 000 Monte Carlo permutations. The $P$-values were adjusted for multiple testing using Holm's method.

Beta diversity analysis was performed using unweighted and weighted UniFrac [25] distances after randomly subsampling from each sample using QIIME's beta_diveristy.py. The sampling depth was set as the read count of the smallest sample. The phylogenetic tree used for the measurement of UniFrac distance was created based on the representative reads (i.e. the most abundant read in each OTU) using SSU-ALIGN (version 0.1.1) [26] and FastTree [27] in QIIME's make_phylogeny.py. Principal coordinate analysis was conducted on the UniFrac distances using QIIME's principal_coordinates.py.

The significance of the compositional difference between two groups of microbiotas was tested using Adonis [28] implemented in QIIME's compare_categories.py with 10,000 permutations. When the number of samples was not large enough to conduct 10,000 permutations, the maximum permutation size was automatically selected by the program. To compensate for UniFrac distance's sensitivity to subsampling, the process of subsampling, measuring UniFrac distances and calculating Adonis $P$-value was repeated 10 times. We used the average of the $P$-values. The $P$-values were adjusted for multiple testing using Holm's method.

Prior to testing the significance of differential abundance of OTUs, OTUs supported by less than a total of 100 reads from samples in the pair of groups under comparison were omitted from analysis. Furthermore, OTUs observed in less than $25 \%$ of the total number of samples under comparison were also removed. These steps were done using QIIME's filter_otus_from_otu_table.py. The statistical significance of the differential abundance of OTUs between sample groups were tested using the DESeq2's negative binomial Wald test [29] implemented in QIIME's differential_abundance.py. False discovery rate was calculated with the Benjamini-Hochberg correction and the cut-off was set at 0.05 . For each OTU that showed significant differential abundance, the sample group with the higher average relative abundance was examined. If any of the samples belonging to this group had no reads for the OTU in question, we did not consider the OTU to be differentially represented. Furthermore, if the maximum relative abundance of the OTU in the lowabundance group was higher than the relative abundance of the same OTU in more than half of the samples of the high-abundance group, the OTU was not considered to be differentially represented.

In some specific cases, we inspected detailed taxonomy of OTUs using homology searches. Homology searches of the representative sequences of OTUs (i.e., full length 
Greengenes sequences or the most abundant read if former is not available) against the NCBI's nucleotide collection $(\mathrm{nr} / \mathrm{nt})$ database were conducted using the online megablast tool $[30,31]$ with the default settings.

\section{Statistical analysis}

Statistical tests were performed using two-tailed Student's $t$-test with $P$ value $<0.05$ as the significance cut-off, unless otherwise stated.

\section{Results}

Mice that lack GK levels in epithelial cells in the upper small intestine are hypersusceptible to DSS-induced colitis

Initially we tried to determine the role of GK in the development of inflammation in the colon. To achieve this, we produced Gankyrin fff mice [8] and crossed them with (i) Villin-Cre and (ii) Cdx2-Cre transgenic mice to generate mice that lack GK levels in their intestinal epithelial cells of (i) the whole small intestine and the colon (Villin-Cre;Gankyrin ${ }^{f / f}$ mice) and (ii) the distal small intestine and the colon (Cdx2-Cre;Gankyrin ${ }^{f / f}$ mice), respectively (Additional file 1: Figure S1). Experimental colitis was induced by treating the mice with $2.5 \%$ DSS for 7 days. The degree of inflammatory cell infiltration into the colon and the epithelial injury were higher in Villin-Cre;Gankyrin ${ }^{f / f}$ mice $(n=6)$ than in Gankyrin ${ }^{f / f}$ control mice $(n=5) \quad(P<0.05$, Fig. 1a and b). Furthermore, we investigated the change in immune response associated with GK-deficiency by analyzing the cytokine levels in the colon. Colonic tissues from Villin-Cre;Gankyrin $^{f / f}$ mice showed an enhanced immune response with high levels of pro-inflammatory cytokines IL-17A, IL-23, and IFN- $\gamma$ (Fig. 1c). In the DSS-induced colitis model, body weight of mice reflects the extent of colonic inflammation [6]. On day 7, a significant difference was seen in body weight between Villin-Cre;Gankyrin ${ }^{f / f}$ and control mice treated with DSS. In contrast, Cdx2-Cre; Gankyrin $^{f / f}$ and control mice showed similar levels of body weight (Fig. 1d). The GK-deficiency in epithelial cells of the upper small bowel, but not in the colon, had augmented the colonic inflammation.

\section{The reduced expression of antimicrobial peptides in GK-deficient intestinal epithelium}

We tried to explore the mechanisms by which GK deletion in the upper small intestine augment DSS-induced colitis. Antimicrobial peptide Defensin is produced by Paneth cells that are mostly located in the upper small bowel. Quantification of the expression of defensin genes (Defa5 and Defa6) in small intestine sections by quantitative PCR with reverse transcription revealed significant decreases of defensin expression in upper small bowel in Villin-Cre;Gankyrin ${ }^{\text {flf }}$ mice, suggesting that GK is at least partially responsible for the transcription of defensins in this part of intestinal tract (Fig. 2a). On treatment with DSS, Defa 5 and Defa6 mRNA expressions were significantly reduced in Villin-Cre;Gankyrin ${ }^{\text {flf }}$ mice compared to that in controls $(P<0.05$, Fig. 2b). Immunohistochemistry confirmed reduced expression of GK in the upper small bowel (jejunum) of Villin-Cre;Gankyrin ${ }^{\text {f/f }}$ mice, but not of Cdx2-Cre;Gankyrin ${ }^{f / f}$ and control mice (Fig. 2c). No difference in the number of Paneth cells was found among GK-deficient and control mice (Fig. 2c). To assess Paneth cell function, the number of granules per cell was counted. As shown in Fig. 2c, there was no difference in the number of granules. Unlike in the colon, no obvious inflammation was found both in Villin-Cre Gankyrin $^{f / f}$ and control mice in the small intestine (Fig. 1, 2a, c, and Additional file 1: Figure S1). This might be explained by the difference in the interaction of microbiota and immune system between the small bowel and the colon [32].

To investigate another possible mechanism by which GK in the upper small bowel affects colitis, we conducted expression profiling of GK-deficient (Villin-Cre; Gankyrin $^{f / \mathcal{S}}$ ) as well as control small intestine sections using whole-genome gene expression arrays. Data analysis revealed changes in the expression of several genes related to the regulation of stem cells. We confirmed the decrease in expression of R-spondin 1 and Wnt5B by quantitative RT-PCR of the GK-deficient small intestine samples relative to control samples (Fig. 2d), which was consistent with previous reports $[8,10,33,34]$. There was no difference in the length of small bowel between Villin-Cre Gankyrin ${ }^{f / f}$ and control mice. At least in this model, Gankyrin deficiency does not affect intestinal regeneration that is one of stem cell functions.

Some patients with Crohn's disease (CD) suffer from colonic involvement, while the disease is restricted to the small intestine in the remaining CD patients. Therefore, we examined whether GK in the upper small bowel contributes to colonic involvement in CD. Tissue samples of the upper small bowel from CD patients with colonic involvement showed a significantly lower level of GK expression than that in the samples from CD patients without colonic involvement $(P<0.05$, Fig. 2 e) .

\section{GK-deficiency causes alterations in bacterial community of the gut}

Host antimicrobial peptides such as defensins exhibit a major influence on the microbial community structure in gut [35]. To understand the relationship between intestinal microbiota and the augmentation of inflammation by the deficiency of GK in the small intestine, $16 \mathrm{~S}$ rRNA gene amplicon analyses were conducted to characterize the caecum and rectum microbiota of $\mathrm{Vil}$ lin-Cre;Gankyrin ${ }^{f / f}$ and Gankyrin ${ }^{f / f}$ control mice. In total, 


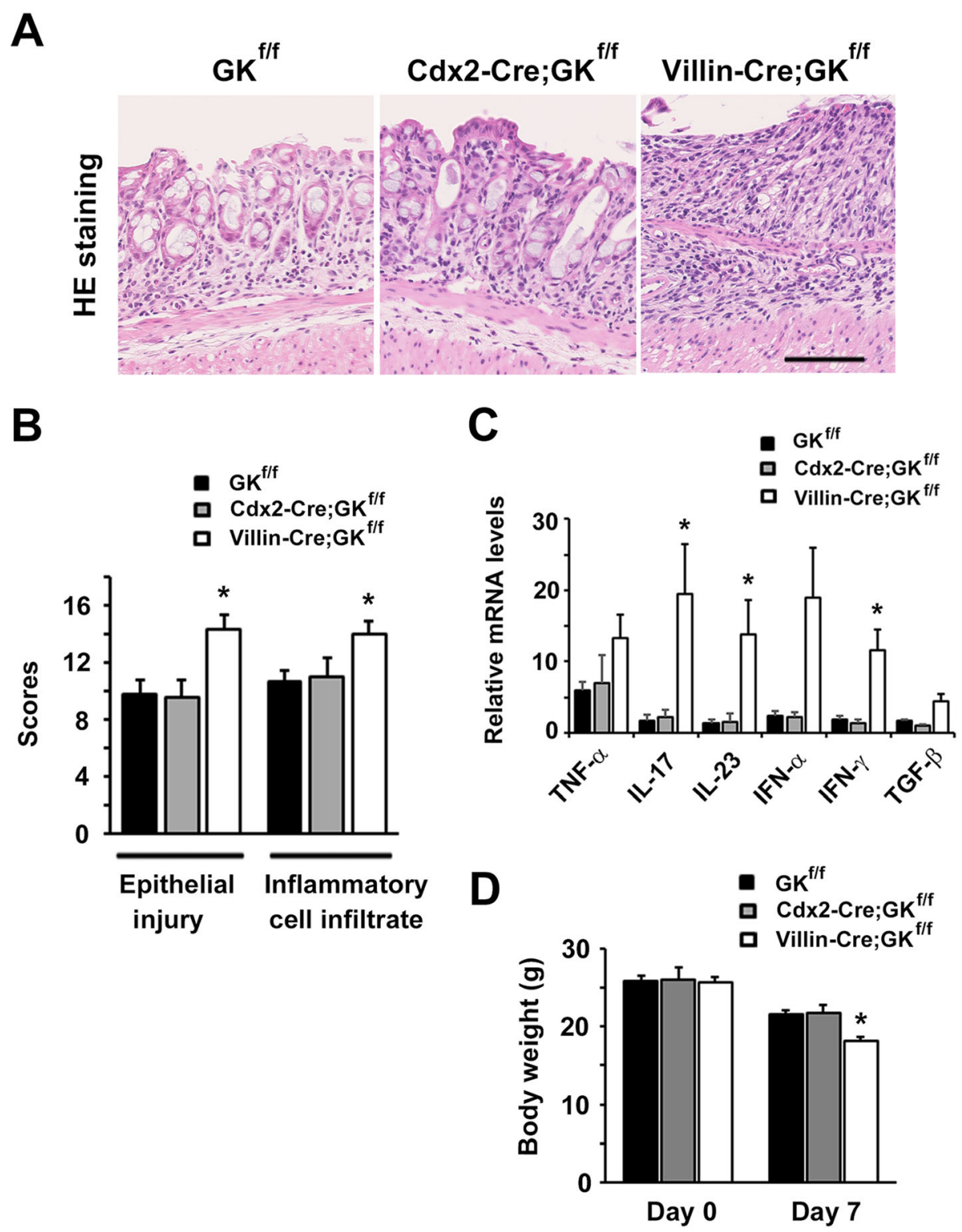

Fig. 1 Mice that lack Gankyrin (GK) levels in epithelial cells in the upper small intestine are hypersusceptible to dextran sodium sulfate (DSS)induced colitis. (A) Representative images of hematoxylin and eosin stained colonic tissues in Gankyrin ${ }^{f / f}\left(G^{f / f}\right)$, Cdx2-Cre; Gankyrin ${ }^{f / f}$ (Cdx2Cre; GK ${ }^{f / f}$ ) and Villin-Cre;Gankyrin ${ }^{f / f}$ (Villin-Cre;GK ${ }^{\mathrm{f} / f}$ ) mice. Cdx2-Cre;GK $K^{\mathrm{f} / \mathrm{f}}$, Villin-Cre;GK ${ }^{\mathrm{flf}}$ and $\mathrm{GK}^{\mathrm{f} / \mathrm{f}}$ mice were administered with $2.5 \%$ DSS in drinking water for 7 days. Scale bar, $100 \mu \mathrm{m}$. (B) Epithelial injury and inflammatory cell infiltration into colonic tissues of GK ${ }^{\mathrm{f} / \mathrm{f}}$, $\mathrm{Cdx2}_{\mathrm{C}}-\mathrm{Cre}_{\mathrm{G}} \mathrm{GK}^{\mathrm{f} / \mathrm{f}}$, and VillinCre; $G K^{\mathrm{f} / \mathrm{f}}$ mice were observed after 7 days of the initiation of DSS administration. $n=6$ per group. (C) RNA was extracted from colonic tissues of DSS-treated Villin-Cre; GK ${ }^{f / f}(n=6), C d \times 2-C r e ; G K{ }^{f / f}(n=4)$, and $\mathrm{GK}^{\mathrm{f} / f}(n=5)$ mice. Relative quantities of mRNA were determined by real-time $\mathrm{qPCR}$ and were normalized to the quantity of $\beta$-actin mRNA. The relative quantity of each mRNA in the untreated colon was assumed to be an arbitrary value of 1.0. Data are represented as mean \pm SEM. (D) Body weight of DSS-treated Villin-Cre;GK $K^{f / f}(n=6), C d \times 2-C r e ; G K K^{f / f}(n=4)$, and GK ${ }^{f / f}$ $(\mathrm{n}=5)$ mice were shown at the time point of day 0 and day 7

we generated 13,442 OTUs, which contained 2,593,734 merged reads (Tables 1 and 2).

Dysbiosis is a state of microbiota which is marked by multiple factors such as a decreased bacterial community diversity and an altered bacterial composition. Dysbiosis is a frequently observed feature in the intestinal microbiota of IBD patients [36]. Consistently, colitis-induced mice showed a lower OTU richness (i.e. the number of OTUs) in comparison with their colitis non-induced counterparts in both caecum and rectum (Fig. 3a). The decreases in OTU richness of the colitis induced samples were statistically significant for both Villin-Cre;Gankyrin ${ }^{f / f}$ and control mice $(P<0.05$ with Holm's correction, Table 3). Shannon diversity index also showed a similar trend, though statistical 

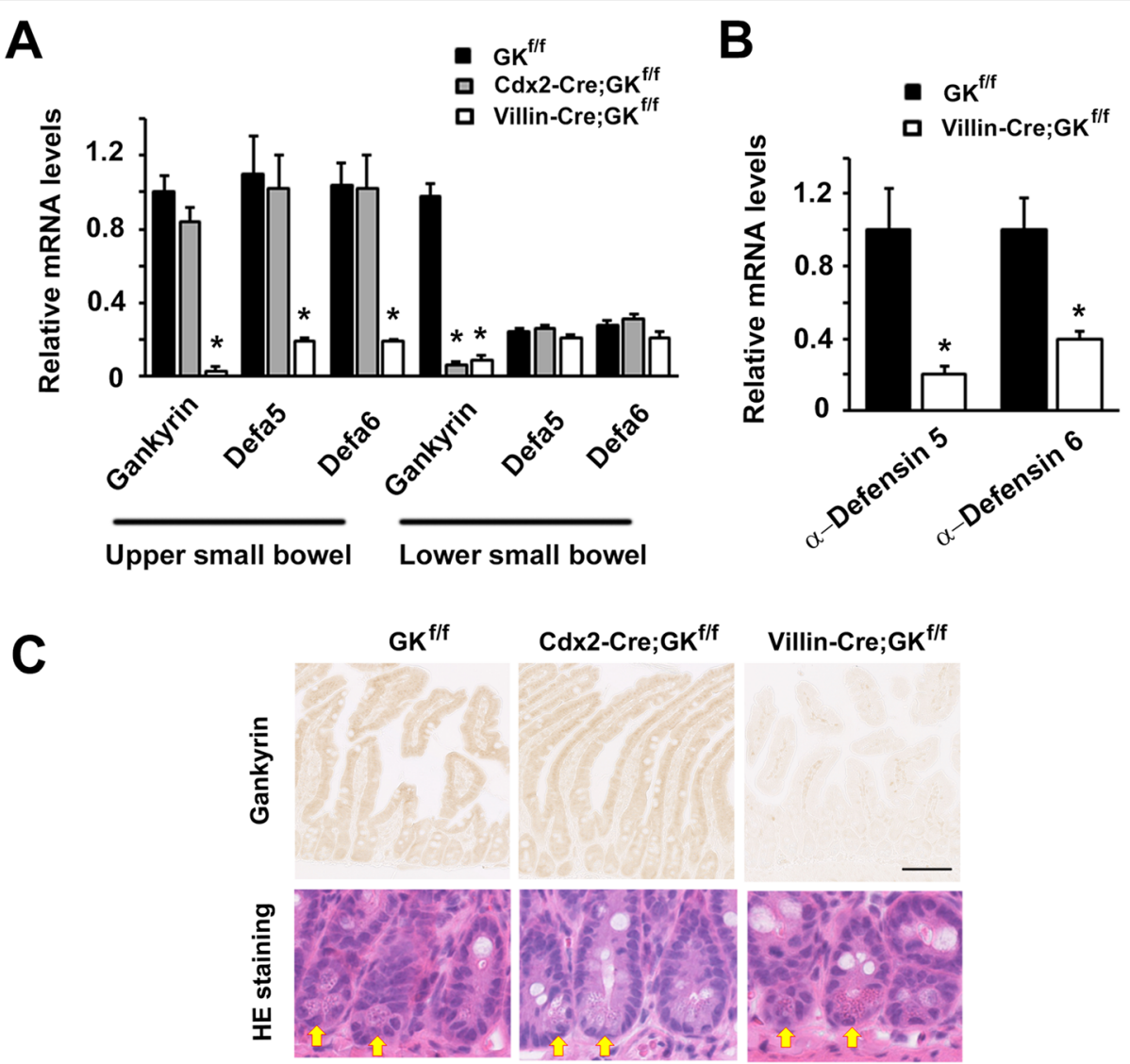

Cdx2-Cre;GK ${ }^{f / f}$

Villin-Cre;GK ${ }^{f / f}$

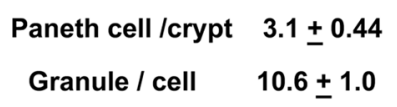

D

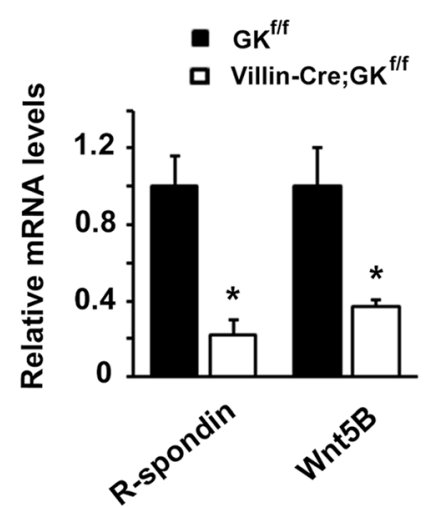

E

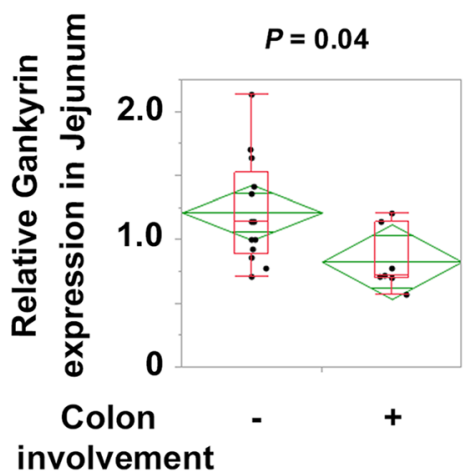

Fig. 2 (See legend on next page.)

significance was observed only between rectum samples of colitis induced and colitis non-induced Villin-Cre;Gankyr$i n^{f / f}$ mice $(P<0.05$ with Holm's correction, Table 3$)$.

To investigate the variation of bacterial composition among the samples, a principal coordinate analysis was conducted based on the unweighted UniFrac distances (Fig. 3b). In the plot, samples from colitis-induced mice and their colitis non-induced counterparts were clearly separated from each other. In both caecum and rectum samples, the compositional difference between these two groups were statistically significant for both Villin-Cre; Gankyrin $^{f / f}$ and control mice (Adonis test, $P<0.05$ with Holm's correction, Table 4). Furthermore, many of the samples from the colitis-induced Villin-Cre;Gankyrin ${ }^{f / f}$ mice were placed apart from the samples of colitisinduced Gankyrin fff control mice in both caecum and 
(See figure on previous page.)

Fig. 2 The reduced expression of antimicrobial peptides in GK-deficient intestinal epithelium. RNA was extracted from (A) the upper small bowel and the lower small bowel tissues of untreated Villin-Cre;Gankyrin ${ }^{f / f}\left(\right.$ Villin-Cre;GK $\left.{ }^{\mathrm{f} / f}\right)$ and Gankyrin ${ }^{\mathrm{f} / f}\left(\mathrm{GK}^{\mathrm{K} / f}\right)$ mice, and (B) the upper small bowel tissues of DSS-treated Villin-Cre;GK $K^{f / f}$ and $G^{f / f}$ mice. The relative quantities of mRNA in all the above samples were determined by real-time $\mathrm{qPCR}$ and were normalized to the quantity of $\beta$-actin mRNA. The relative quantity of each mRNA in the untreated small intestine samples was assumed to be an arbitrary value of 1.0. Data are represented as mean $\pm \operatorname{SEM}(n=3)$. (C) Immunohistochemistry using anti-GK antibody and Hematoxylin \& Eosin (HE) staining were performed on the upper small bowel samples of Villin-Cre; GK ${ }^{\mathrm{f} / \mathrm{f}}$, Cdx2-Cre; $\mathrm{GK}^{\mathrm{f} / \mathrm{f}}$, and $\mathrm{GK}^{\mathrm{f} / \mathrm{f}}$ mice at day 7 . Arrows indicate Paneth cells at the base of the intestinal glands. The numbers of Paneth cells per crypt and granules per one Paneth cell were shown. (D) RNA was extracted from the upper small bowel tissues of untreated Villin-Cre; $\mathrm{GK}^{\mathrm{flf}}$ and $\mathrm{GK}^{\mathrm{f} / \mathrm{f}}$ mice. The relative quantities of mRNA in all the above samples were determined by real-time qPCR and were normalized to the quantity of $\beta$-actin mRNA. The relative quantity of each mRNA in the untreated small intestine samples was assumed to be an arbitrary value of 1.0. Data are represented as mean \pm SEM $(n=3)$. (E) RNA was extracted from the upper small bowel (jejunum) tissues of patients suffering from Crohn's disease (i) with $(n=7)$ and (ii) without $(n=13)$ colonic involvement. The relative quantities of GK mRNA were determined by real-time qPCR and were normalized to the quantity of $\beta$-actin mRNA. The relative quantity of each mRNA in the untreated small intestine samples was assumed to be an arbitrary value of 1.0. ${ }^{*}$ indicates a statistically significant difference (Student's $t$-test, two tailed, $P<0.05$ )

rectum (Fig. 3b). The compositional difference between these two groups was statistically significant in caecum samples but not for rectum samples (Adonis test, $P<$ 0.05 with Holm's correction, Table 4). Samples from colitis non-induced Villin-Cre;Gankyrin ${ }^{f / f}$ mice and colitis non-induced control mice were spatially closely located in the principal coordinate plots (Fig. 3b). The two groups did not significantly differ from each other (Adonis test, $P>0.05$ ) for caecum and rectum samples, though sample size was small.

We also performed a principal coordinate analysis based on weighted UniFrac distances between all samples (Additional file 2: Figure S2). In the plots, colitis-induced mice and their colitis non-induced counterparts were once again separated, though statistical significance was observed only in rectum samples of Villin-Cre;Gankyrin ${ }^{\text {fff }}$ mice (Adonis test, $P<0.05$ with Holm's correction, Additional file 9: Table S1). A less enhanced separation between Villin-Cre;Gankyrin ${ }^{f / f}$ and control mice for colitis induced cases, and a clearer separation for colitis noninduced cases was observed (Additional file 2: Figure S2). Statistical significance was not observed for both cases (Adonis test, $P>0.05$, Additional file 9: Table S1).

\section{Intestinal bacteria showing differential abundance in association with the GK knockout}

To better understand the effect of GK deficiency on the bacterial composition of intestinal microbiota, samples were compared to identify OTUs showing differential abundance. First comparisons were conducted between

Table 1 Number of sequence reads derived from caecum samples

\begin{tabular}{|c|c|c|c|c|}
\hline Treatment & Mice & Pairs of raw reads & Merged reads & Reads in OTUs \\
\hline \multirow[t]{6}{*}{ Untreated } & \multirow[t]{3}{*}{$\mathrm{GK}^{\mathrm{f} / \mathrm{f}}$} & 45,733 & 37,945 & 19,828 \\
\hline & & 153,593 & 134,494 & 69,296 \\
\hline & & 146,664 & 127,381 & 67,536 \\
\hline & \multirow[t]{3}{*}{ Villin-Cre;GK ${ }^{f / f}$} & 138,984 & 116,816 & 52,554 \\
\hline & & 205,983 & 173,395 & 81,165 \\
\hline & & 174,592 & 151,042 & 77,839 \\
\hline \multirow[t]{11}{*}{ DSS-treated } & \multirow[t]{5}{*}{$\mathrm{GK}^{\mathrm{f} / \mathrm{f}}$} & 111,473 & 89,718 & 67,125 \\
\hline & & 82,429 & 62,535 & 47,108 \\
\hline & & 86,110 & 82,334 & 47,630 \\
\hline & & 322,471 & 309,961 & 165,065 \\
\hline & & 256,380 & 245,169 & 142,468 \\
\hline & \multirow[t]{6}{*}{ Villin-Cre;GK ${ }^{f / f}$} & 170,690 & 147,242 & 85,464 \\
\hline & & 109,655 & 96,143 & 59,270 \\
\hline & & 118,801 & 104,575 & 48,277 \\
\hline & & 215,726 & 185,793 & 101,640 \\
\hline & & 94,345 & 90,886 & 54,584 \\
\hline & & 424,553 & 403,679 & 265,611 \\
\hline Total & & $2,858,182$ & $2,559,108$ & $1,452,460$ \\
\hline
\end{tabular}


Table 2 Number of sequence reads derived from rectum samples

\begin{tabular}{|c|c|c|c|c|}
\hline Treatment & Mice & Pairs of raw reads & Merged reads & Reads in OTUs \\
\hline \multirow[t]{6}{*}{ Untreated } & $\mathrm{GK}^{\mathrm{f} / \mathrm{f}}$ & 108,219 & 93,088 & 57,378 \\
\hline & & 119,144 & 104,912 & 64,302 \\
\hline & & 132,543 & 116,104 & 56,395 \\
\hline & Villin-Cre;GK & 166,120 & 141,459 & 71,663 \\
\hline & & 177,145 & 150,617 & 88,569 \\
\hline & & 153,489 & 130,854 & 65,596 \\
\hline \multirow[t]{12}{*}{ DSS-treated } & $\mathrm{GK}^{\mathrm{f} / \mathrm{f}}$ & 158,088 & 140,514 & 82,872 \\
\hline & & 162,429 & 146,540 & 85,937 \\
\hline & & 146,585 & 129,640 & 72,774 \\
\hline & & 96,962 & 91,832 & 66,395 \\
\hline & & 82,258 & 76,967 & 51,798 \\
\hline & & 67,262 & 64,477 & 47,707 \\
\hline & Villin-Cre;GK/ff & 146,398 & 129,707 & 76,916 \\
\hline & & 149,141 & 133,210 & 70,468 \\
\hline & & 129,604 & 117,091 & 60,096 \\
\hline & & 64,784 & 61,357 & 40,852 \\
\hline & & 58,762 & 55,256 & 41,103 \\
\hline & & 69,251 & 64,639 & 40,453 \\
\hline Total & & $2,188,184$ & $1,948,264$ & $1,141,274$ \\
\hline
\end{tabular}

colitis-induced Villin-Cre;Gankyrin fff mice and colitisinduced Gankyrin fff control mice for both caecum and rectum samples. The comparisons revealed 39 OTUs in caecum and 25 OTUs in rectum with differential abundance between the groups of samples (see Methods for the criteria for differential abundance) (Figs. 4 and 5). OTUs over-represented (i.e. higher relative abundance) in VillinCre;Gankyrin ${ }^{\text {fff }}$ mice (caecum and/or rectum samples) belonged to Bilophila (1 OTU, caecum and rectum), Helicobacteraceae (3 OTUs, rectum), Bacteroidales S24-7 (10 OTUs, caecum and rectum), Clostridiales (15 OTUs, caecum and rectum), and Erysipelotrichaceae (1 OTU, caecum and rectum). BLAST searches for the three OTUs belonging to Helicobacteraceae revealed that the representative sequence of OTU-206538 was aligned with the 16S rRNA gene of Helicobacter japonicum (NR_149210.1 and EF373968.1) with 100\% sequence identify. The other two OTUs were also aligned with the $16 \mathrm{~S}$ rRNA gene sequences of Helicobacter species with $100 \%$ sequence identity (GU902718.1 and AB693139.1 for OTU-675509 and OTU2564049, respectively). OTU-206538 (Helicobacter japonicum) showed a notably high relative abundance (average $18.8 \%$ ) in the rectum samples of Villin-Cre;Gankyrin ${ }^{\text {ff }}$ mice (Fig. 5). OTUs under-represented (i.e. lower relative abundance) in caecum and/or rectum samples of colitis-induced Villin-Cre;Gankyrin ${ }^{f / f}$ mice belonged to Adlercreutzia (1 OTU, caecum), CW040 F16 (1 OTU, caecum), and Clostridiales (23 OTUs, caecum and rectum).
Next, we compared microbiotas of colitis non-induced Villin-Cre;Gankyrin ${ }^{f / f}$ and colitis non-induced control mice. This comparison revealed 91 OTUs in caecum and 86 OTUs in rectum with differential abundance between the two mice groups (see Methods for the criteria for differential abundance) (Additional file 3: Figure S3, Additional file 4: Figure S4, Additional file 5: Figure S5, Additional file 6: Figure S6). OTUs that were overrepresented in caecum and/or rectum samples from $\mathrm{Vil}$ lin-Cre;Gankyrin ${ }^{f / f}$ mice belonged to Coriobacteriaceae (1 OTU, rectum), Bacteroidales (32 OTUs, caecum and rectum), Lactobacillus reuteri (1 OTU, rectum), Turicibacter (1 OTU, caecum and rectum), Clostridiales (29 OTUs, caecum and rectum), and Allobaculum (1 OTU, caecum and rectum). OTUs that were underrepresented in caecum and/or rectum samples of VillinCre;Gankyrin ${ }^{f / f}$ mice belonged to Bacteroidales (3 OTUs, caecum and rectum), Mucispirillum schaedleri (1 OTU, rectum), Clostridiales (51 OTUs, caecum and rectum), Alphaproteobacteria RF32 (1 OTU, caecum), and Desulfovibrionaceae (2 OTUs, rectum).

\section{Elevated inflammation observed in Villin-Cre;Gankyrin ${ }^{f / f}$ mice is reduced through co-housing}

To have insight into whether the enhanced susceptibility of Villin-Cre;Gankyrin ${ }^{f / f}$ mice to DSS-induced colitis is associated with intestinal microbiota, we performed a co-housing experiment between Villin-Cre;Gankyrin f/f 
A
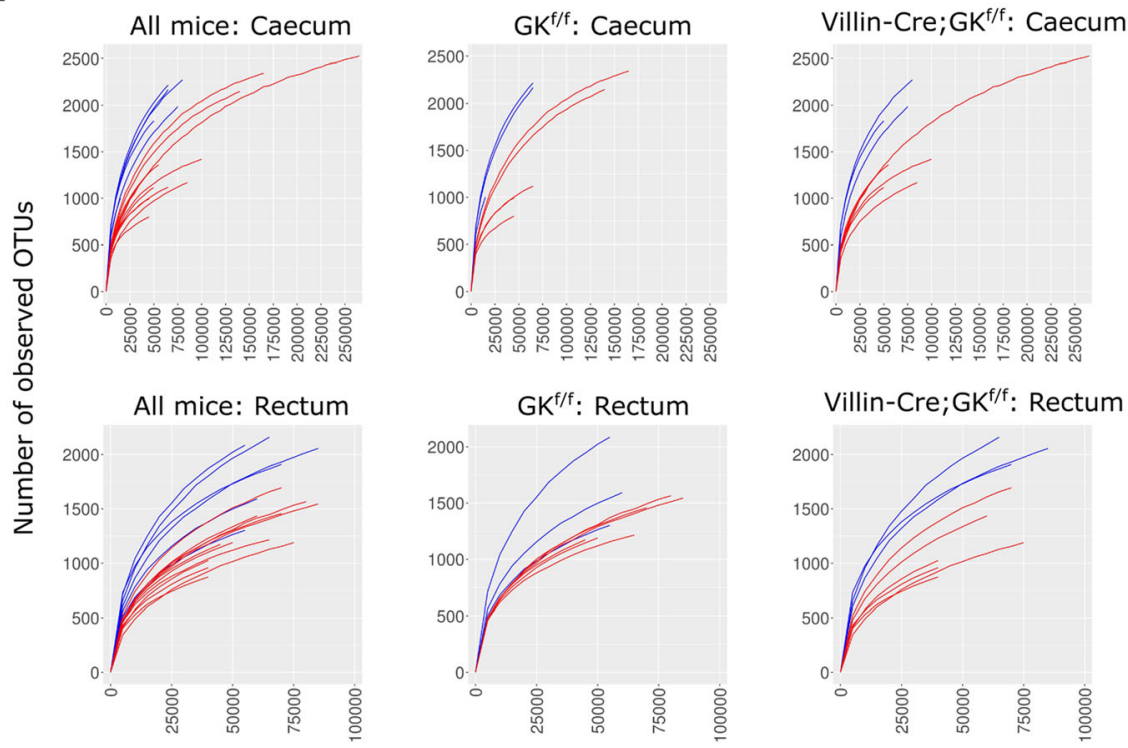

Villin-Cre;GK ${ }^{f / f}$ : Rectum

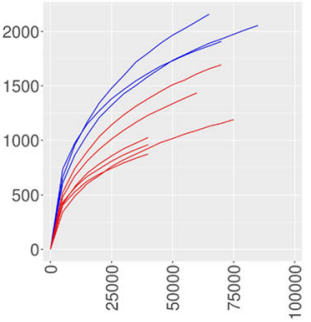

B

Rarefaction depth

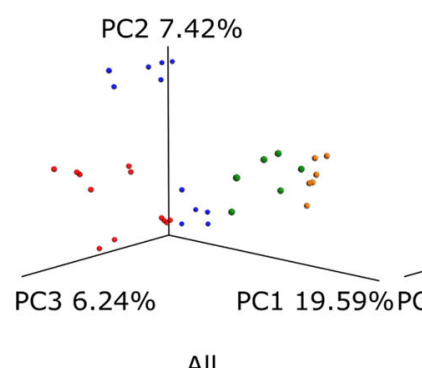

PC2 $7.42 \%$
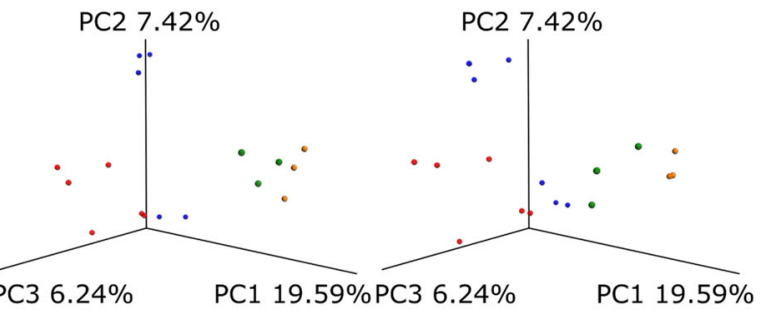

All

Caecum

Rectum

Fig. 3 Comparison of intestinal microbiotas between samples. (A) The numbers of operational taxonomy units (OTUs) at different rarefaction depth are plotted for colitis non-induced mice (blue) and colitis induced mice (red). (B) Principal coordinate analyses of samples based on bacterial operational taxonomic unit (OTU) compositions. Compositional variations were measured by unweighted UniFrac distances. The color codes for the samples are, green: colitis non-induced Gankyrin ${ }^{f / f}$ control mice, orange: colitis non-induced Villin-Cre;Gankyrin ${ }^{f / f}$ mice, blue: colitisinduced Gankyrin ${ }^{f / f}$ control mice, red: colitis-induced Villin-Cre;Gankyrin ${ }^{\text {f/f }}$ mice

Table 3 Richness and Shannon diversity estimates of intestinal microbiota

\begin{tabular}{|c|c|c|c|c|c|c|c|c|c|}
\hline \multirow[t]{2}{*}{ Part } & \multirow[t]{2}{*}{ DSS } & \multirow[t]{2}{*}{ Mice } & \multirow[t]{2}{*}{$\mathrm{n}$} & \multicolumn{3}{|c|}{ Observed amount of OTUs } & \multicolumn{3}{|c|}{ Shannon diversity } \\
\hline & & & & Mean & Max & Min & Mean & Max & Min \\
\hline \multirow[t]{4}{*}{ Caecum } & - & $\mathrm{GK}^{\mathrm{f} / \mathrm{f}}$ & 3 & 1286.0 & 1392.1 & 1124.0 & 6.60 & 6.93 & 6.40 \\
\hline & + & $\mathrm{GK}^{\mathrm{f} / \mathrm{f}}$ & 5 & $833.4^{*}$ & 1031.9 & 630.1 & 5.91 & 6.60 & 5.23 \\
\hline & - & Villin-Cre;GK/f & 3 & 1277.7 & 1354.4 & 1161.5 & 6.50 & 6.97 & 5.63 \\
\hline & + & Villin-Cre;GK/ff & 6 & $830.2^{*}$ & 914.0 & 685.3 & 5.70 & 6.36 & 5.16 \\
\hline \multirow[t]{4}{*}{ Rectum } & - & $\mathrm{GK}^{\mathrm{f} / \mathrm{f}}$ & 3 & 1123.2 & 1412.6 & 905.5 & 6.33 & 6.80 & 5.83 \\
\hline & + & $\mathrm{GK}^{\mathrm{f} / \mathrm{f}}$ & 6 & $870.7^{*}$ & 904.8 & 811.9 & 6.04 & 6.36 & 5.66 \\
\hline & - & Villin-Cre;GK ${ }^{\mathrm{f} / f}$ & 3 & 1261.8 & 1332.8 & 1188.6 & 7.09 & 7.99 & 6.52 \\
\hline & + & Villin-Cre;GK/ff & 6 & $801.2^{*}$ & 1031.0 & 673.8 & $5.77^{*}$ & 6.31 & 4.63 \\
\hline
\end{tabular}


Table 4 The corrected $P$-values and effect sizes reported by the Adonis test when performed on unweighted unifrac distances between sample groups

\begin{tabular}{|c|c|c|c|c|c|c|}
\hline \multirow[t]{3}{*}{ Part } & \multicolumn{4}{|c|}{ Compared mice groups } & \multirow[t]{3}{*}{$P$-value } & \multirow[t]{3}{*}{ Effect size } \\
\hline & \multicolumn{2}{|c|}{ Group A } & \multicolumn{2}{|c|}{ Group B } & & \\
\hline & DSS & Mice & DSS & Mice & & \\
\hline \multirow[t]{4}{*}{ Caecum } & - & $\mathrm{GK}^{\mathrm{f} / \mathrm{f}}$ & - & Villin-Cre;GK/f/f & 0.1000 & 0.32 \\
\hline & + & $\mathrm{GK}^{\mathrm{f} / \mathrm{f}}$ & + & Villin-Cre;GK ${ }^{f / f}$ & $0.0449 *$ & 0.15 \\
\hline & - & $\mathrm{GK}^{\mathrm{f} / \mathrm{f}}$ & + & $\mathrm{GK}^{\mathrm{f} / \mathrm{f}}$ & $0.0449 *$ & 0.36 \\
\hline & - & Villin-Cre;GK $K^{f / f}$ & + & Villin-Cre;GK/f/f & $0.0449^{*}$ & 0.30 \\
\hline \multirow[t]{4}{*}{ Rectum } & - & $\mathrm{GK}^{\mathrm{f} / \mathrm{f}}$ & - & Villin-Cre;GK ${ }^{f / f}$ & 0.1782 & 0.32 \\
\hline & + & $\mathrm{GK}^{\mathrm{f} / \mathrm{f}}$ & + & Villin-Cre;GK ${ }^{\mathrm{f} / \mathrm{f}}$ & 0.1782 & 0.11 \\
\hline & - & $\mathrm{GK}^{\mathrm{f} / \mathrm{f}}$ & + & $\mathrm{GK}^{\mathrm{f} / \mathrm{f}}$ & $0.0478^{*}$ & 0.30 \\
\hline & - & Villin-Cre;GK/ff & + & Villin-Cre;GK ${ }^{f / f}$ & $0.0478^{*}$ & 0.29 \\
\hline
\end{tabular}

indicates a statistical significance ( $P$-value is adjusted by Holm's correction; $P<0.05$ )

and control mice. Villin-Cre;Gankyrin fff and control mice were co-housed for 21 days before being challenged with DSS for 7 days. In this case, elevated inflammatory cell infiltration into the colon was not observed in Villin-Cre;Gankyrin ${ }^{f / f}(P>0.05$; Fig. 6a and b).

Microbiota analysis was also conducted by adding the $16 \mathrm{~S}$ amplicon data from co-housed mice (Additional file 10: Table S2). Contrary to our expectation, the average OTU richness was $50 \%$ higher in Villin-Cre;Gankyrin fff mice in comparison with Gankyrin ${ }^{f / f}$ control mice; the average Shannon diversity index was 30\% higher in VillinCre;Gankyrin ${ }^{f / f}$ mice. However, these differences were not statistically significant $(P>0.05$, Additional file 11 : Table S3). Principal component analysis based on both weighted UniFrac and unweighted UniFrac distances revealed separation of the two groups in space (Additional file 7: Figure S7), though these compositional differences were not statistically significant (Adonis test, $P>0.05$ ). Comparison of the relative abundances of OTUs revealed 10 OTUs with differential abundance between the Villin-Cre;Gankyrin $^{f / f}$ and control mice (Additional file 8: Figure S8). OTUs over-represented in Villin-Cre;Gankyrin ${ }^{f / f}$ mice belonged to Clostridiales (3 OTUs). OTUs underrepresented in Villin-Cre;Gankyrin ${ }^{\text {flf }}$ mice belonged to Helicobacteraceae (4 OTUs), Clostridiales (2 OTUs), and Enterobacteriaceae (1 OTU). The representative sequences of the four Helicobacteraceae OTUs were compared against the NCBI's nr/nt. None of them could be assigned to Helicobacter japonicum.

\section{Discussion}

Previously, we found that GK upregulates the expression of TNF- $\alpha$ and IL-17 in lamina propria and Kupffer cells, which was accompanied by the enhanced MAP kinase level and expansion of cancer stem cells, subsequently leading to inflammation-associated carcinogenesis $[8,10]$. Regarding the mechanisms of GK-induced inflammatory responses in myeloid cells, we showed that the molecular interaction between GK and Src homology 2-containing protein tyrosine phosphatase-1 (SHP-1) leads to the activation of signal transducer and activator of transcription-3 (STAT3) and the secretion of pro-inflammatory cytokines $[8,10]$. In this study, we generated two kinds of conditional GK-deficient mice: Villin-Cre;Gankyrin ${ }^{f / f}$ and Cdx2-Cre;Gankyrin ${ }^{f / f}$ mice. GK was deleted in the epithelial cells of (i) complete small intestine and colon for Villin-Cre;Gankyrin ${ }^{f / f}$ mice and (ii) distal small intestine and colon for Cdx2-Cre;Gankyrin fff mice. Unexpectedly, the development of colitis that was induced by DSS treatment was significantly augmented in the colon of Villin-Cre;Gankyrin ${ }^{f / f}$ mice as compared to that of control mice. In contrast, colitis that was induced by DSS treatment was comparable between $\mathrm{Cdx} 2-\mathrm{Cre}$;Gankyrin ${ }^{f / f}$ and control mice. Therefore, similar to nuclear factor $-\kappa B$ (NF-кB) signaling [37-39], GK regulates inflammatory response in a conditional- and tissue-dependent manner.

Comparisons of gut microbiota between colitisinduced Villin-Cre;Gankyrin ${ }^{f / f}$ and colitis-induced control mice revealed 39 OTUs in caecum and 25 OTUs in rectum with differential abundance between the two groups of samples. Of these, two OTUs overrepresented in colitis-induced Villin-Cre;Gankyrin ${ }^{f / f}$ mice appeared to be related to the enhanced state of inflammation: Helicobacter japonicum (1 OTU) and Bilophila spp. (1 OTU). Helicobacter japonicum is a recently isolated species shown to cause augmented inflammation in IL-10 deficient mice through the upregulation of the inflammatory cytokines, inducible nitric oxide synthase (iNOS), IFN- $\gamma$, IL-17A, and TNF- $\alpha$ [40]. Interestingly, this is consistent with the elevated expression of IFN- $\gamma$ and IL-17A that we observed in colitis-induced VillinCre;Gankyrin ${ }^{f / f}$ mice, which showed augmented inflammation. In addition, two OTUs belonging to Helicobacter spp. were over-represented in colitis-induced VillinCre;Gankyrin ${ }^{f / f}$ mice. Enterohepatic Helicobacter species (EHS) are known to colonize the intestine and are also associated with IBD [41]. In fact, the elevated abundance of EHS in IBD patients in comparison to healthy subjects was revealed by a meta-analysis based on 14 clinical studies [41]. In addition, $H$. pylori known to be associated with the attenuation of IBD [42] was not detected in any of the samples examined in this study. Therefore, some of these Helicobacter OTUs may have contributed to the augmented inflammation in colitis-induced VillinCre;Gankyrin ${ }^{f / f}$ mice. Bilophila includes B. wadsworthia, a sulfate reducing bacterium which produces $\mathrm{H}_{2} \mathrm{~S}$ [43]. $\mathrm{H}_{2} \mathrm{~S}$ has been experimentally proven to promote intestinal inflammation when administered in amounts exceeding the detoxifying capacity of colonocytes in rats $[44,45]$. Overall, the deficiency of GK in the small intestine during induction of colitis have led to the increased 


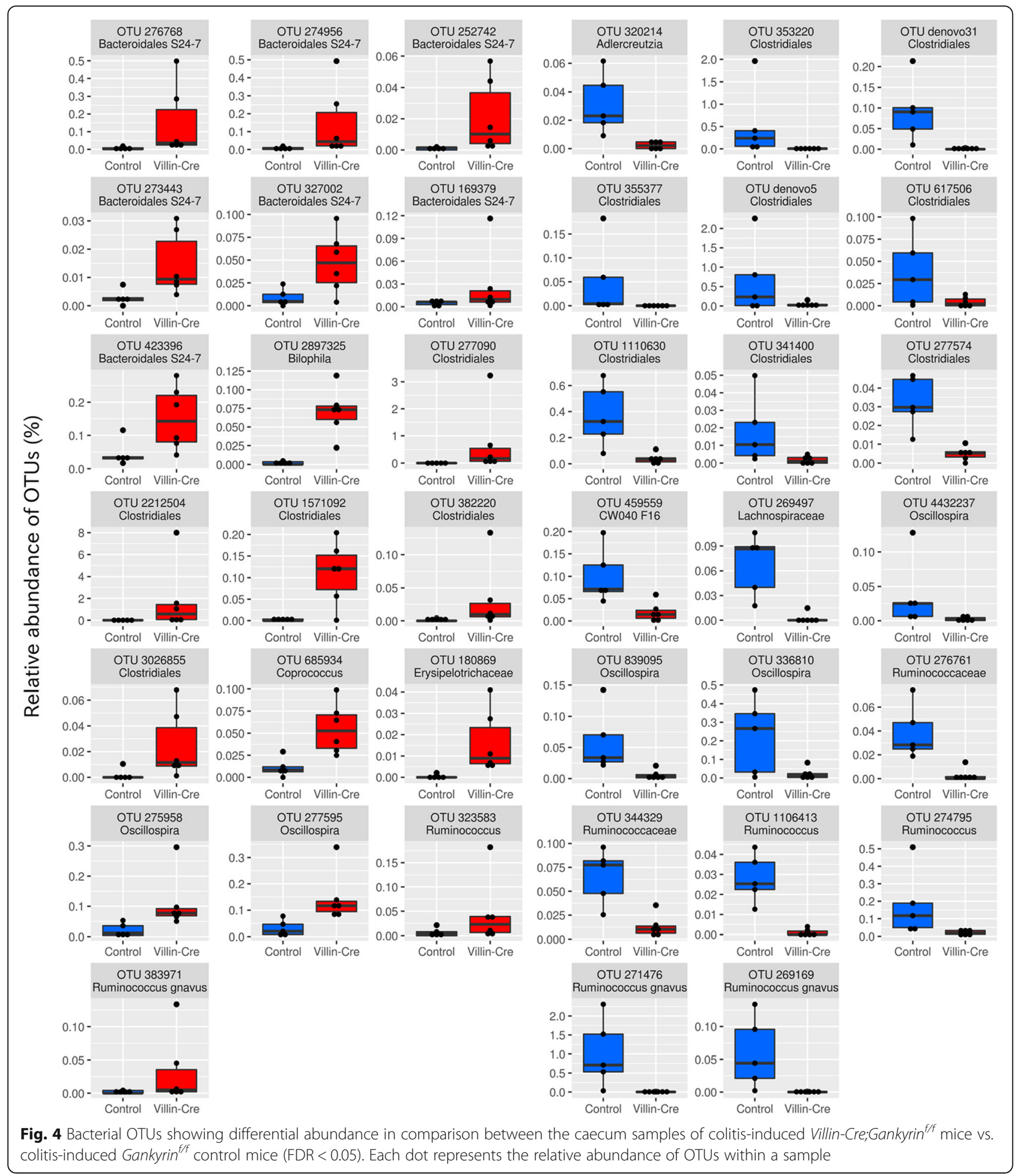

colonization of bacterial species potentially relevant to inflammation.

Between colitis non-induced Villin-Cre;Gankyrin ${ }^{f / f}$ mice and colitis non-induced control mice, numerous OTUs (91 OTUs in caecum and 86 OTUs in rectum) were differentially abundant. It is tempting to speculate that these differences is due to the reduced expression of defensin in the upper small bowel of Villin-Cre;Gankyr$i n^{f f f}$ mice. It is notable that none of the differentially abundant OTUs between the two groups belonged to Helicobacter japonicum nor Bilophila spp. Therefore, the distinct composition of intestinal microbiome in 


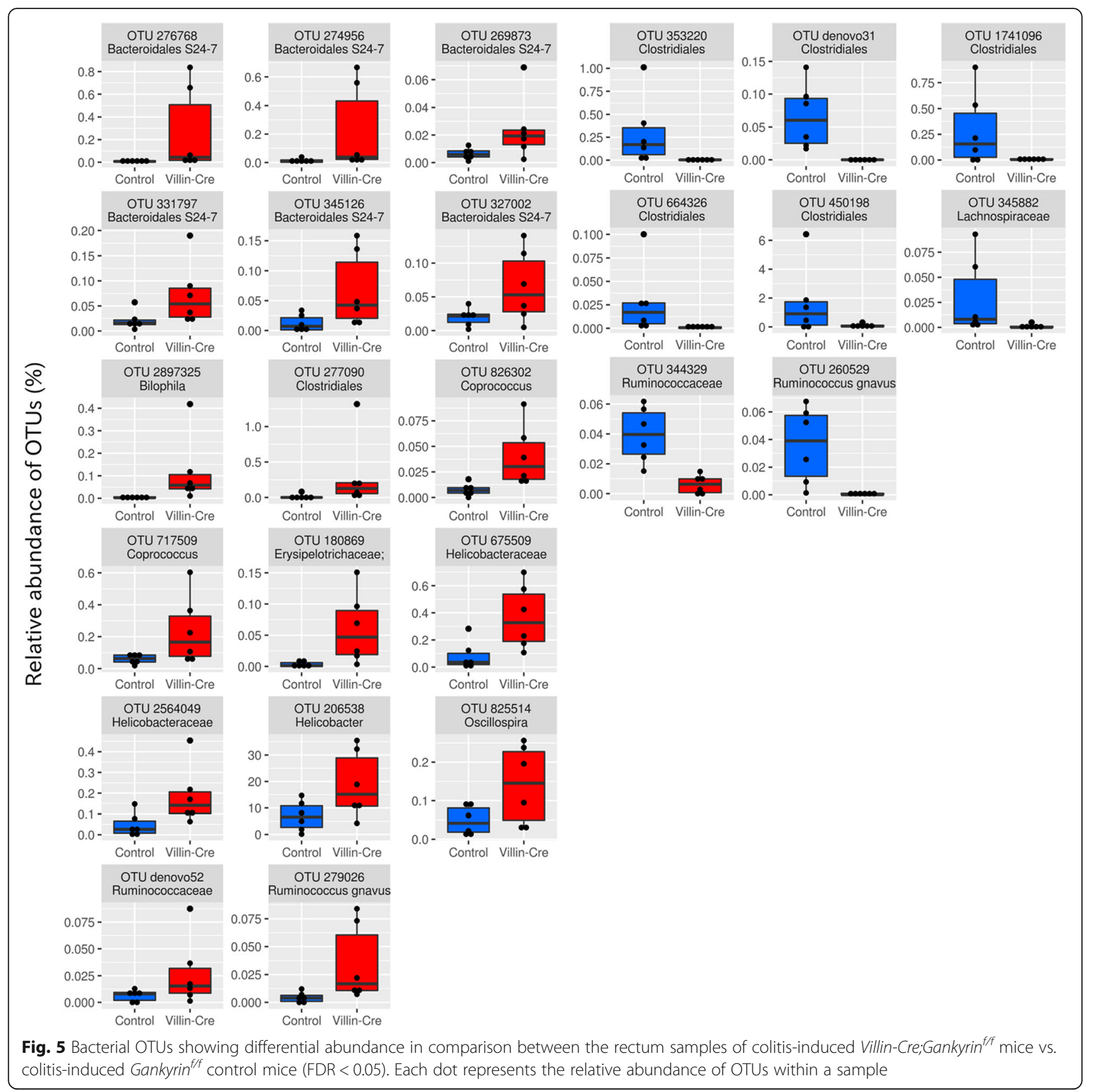

colitis-induced Villin-Cre;Gankyrin ${ }^{f / f}$ mice cannot be explained solely by the decreased defensin production. It appears that a shift in bacterial community was further induced upon colitis induction. Such an alteration of community composition may be a result of intricate dynamic interactions among intestinal inflammation, microbiome and a decreased level of defensin.

In the mice co-housing experiment, there were several OTUs with significant differential abundances. Therefore, contrary to our expectation, co-housing did not lead to similar intestinal microbiomes between Villin-Cre;Gankyrin fff and control mice. However, these differentially abundant
OTUs provide some insights into the lowered inflammation for Villin-Cre;Gankyrif fff mice. There was no differentially abundant OTU related to Helicobacter japonicum or Bilophila spp. This reinforces our idea that over-representation of the OTUs related to Helicobacter japonicum and Bilophila spp. were involved in the elevated intestinal inflammation in the non-cohoused colitis-induced Villin-Cre; Gankyrin ${ }^{f f f}$ mice.

Epithelial antimicrobial peptides such as defensins act as important components within a complex gut barrier defense system. Deficiency of $\alpha$-defensin in Paneth cells is known to cause significant changes in the composition 
A

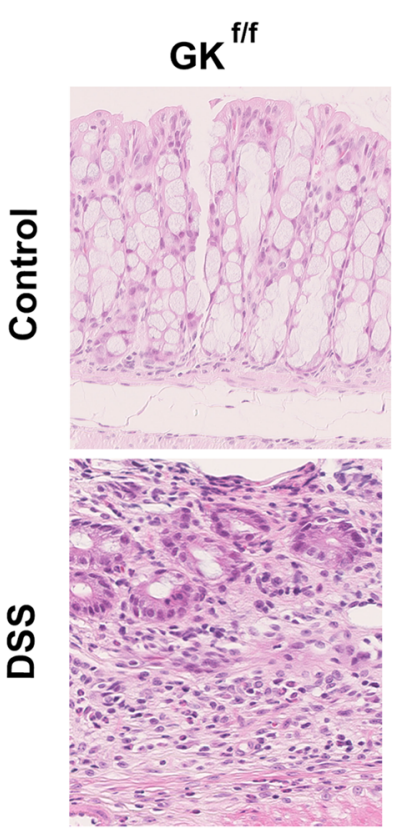

Villin-Cre;GK ${ }^{\mathrm{flf}}$
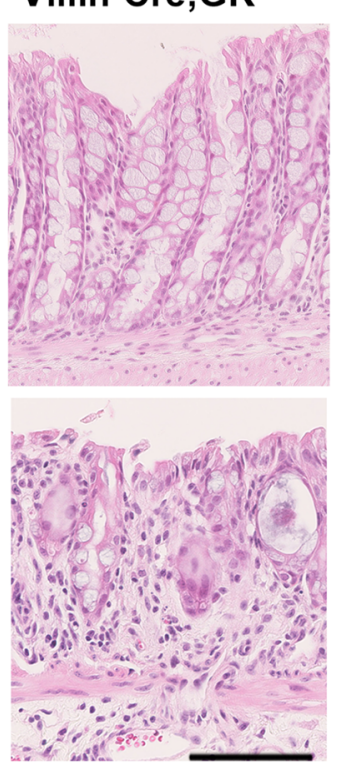

B

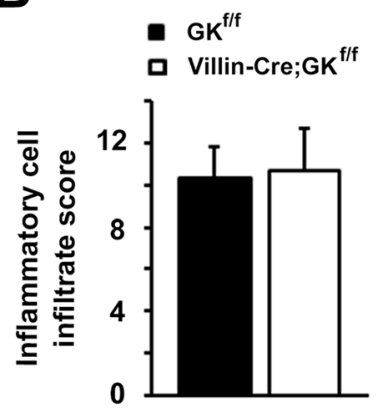

Cohouse experiment

Fig. 6 The degree of inflammatory cell infiltration was not elevated in Villin-Cre;Gankyrin ${ }^{f / f}$ mice when co-housed with control mice. (A) Representative images of hematoxylin \& eosin-stained colonic tissues in co-housed Gankyrin ${ }^{f / f}\left(G^{f / f}\right)$ and Villin-Cre; Gankyrin ${ }^{f / f}\left(\right.$ Villin-Cre;GK $\left.{ }^{f / f}\right)$ mice. Villin-Cre; $G K^{\mathrm{f} / \mathrm{f}}$ and $\mathrm{GK}^{\mathrm{f} / \mathrm{f}}$ mice were administered with dextran sodium sulfate (DSS) in drinking water for 7 days. Scale bar, $100 \mu \mathrm{m}$. (B) Inflammatory cell infiltration into colonic tissues of co-housed $\mathrm{GK}^{\mathrm{f} / \mathrm{f}}$ and Villin-Cre; $\mathrm{GK}^{\mathrm{f} / \mathrm{f}}$ mice after 7 days of the initiation of DSS administration. $\mathrm{n}=3$ per group. ${ }^{*}$ indicates a statistically significant difference (Student's $t$-test, two tailed, $P<0.05$ )

of gut microbiota, suggesting an essential interaction between the gut microbiota and the host [46]. Our results suggest a pivotal role played by the upper small bowel in the development of colitis. Paneth cells, a major site of defensin production, are mainly located in the upper small bowel. Therefore, it can be hypothesized that lowered expression of GK by Paneth cells may affect defensin production, which in turn induces gut microbiota alteration and the development of colitis. However, whether GK is the direct regulator of $\alpha$-defensin remains to be investigated. Further studies on Paneth cellspecific GK-deficient mice are necessary to test our hypothesis.

\section{Conclusions}

The enhanced activation of GK induced by chronic inflammation promotes inflammatory response and inflammation-associated carcinogenesis [8, 10]. However, our findings illustrate the possibility of GK being involved in a negative regulation of inflammation. We propose that this negative regulatory mechanism would function by augmenting the host defense rather than by inhibiting inflammation. The augmented production of defensins and the alterations in microbiota by the small intestinal cells that express GK, may provide a compensatory mechanism that attenuates robust activation of inflammation. These results highlight the distinct site dependence of the pro- and anti-inflammatory functions of GK and provide important insights into the pathogenesis of IBD.

\section{Supplementary information}

Supplementary information accompanies this paper at https://doi.org/10. 1186/s12876-019-1156-0.

Additional file 1: Figure S1. Representative images of

immunohistochemical detection of Gankyrin (GK) are shown in Gankyrin ${ }^{\text {fl/ }}$ $\left(G^{f / f}\right), C d \times 2-C r e ; G a n k y r i n^{f / f}(C d \times 2-C r e ; G K / /)$ and Villin-Cre;Gankyrin ${ }^{f / f}$ (VillinCre;GK $K^{\mathrm{f} / \mathrm{f}}$ ) mice. Scale bar, $100 \mu \mathrm{m}$

Additional file 2: Figure S2. Principal coordinate analysis of weighted UniFrac distance between all samples based on bacterial operational taxonomic units (OTUs). The color codes for the samples are, green: colitis non-induced Gankyrin ${ }^{\mathrm{f} / f}$ control mice, orange: colitis non-induced Villin-Cre;Gankyrin ${ }^{f / f}$ mice, blue: colitis-induced Gankyrin ${ }^{f / f}$ control mice, red: colitis-induced Villin-Cre;Gankyrin ${ }^{f / f}$ mice.

Additional file 3: Figure S3. Bacterial OTUs under-represented in caecum samples of colitis non-induced Villin-Cre;Gankyrin ${ }^{f / f}$ mice in comparison with caecum samples of colitis non-induced Gankyrin ${ }^{\text {fff }}$ control mice (FDR $<0.05)$. Each dot represents the relative abundance of OTUs within each sample.

Additional file 4: Figure S4. Bacterial OTUs over-represented in caecum samples of colitis non-induced Villin-Cre;Gankyrin ${ }^{\text {fff }}$ mice in comparison with caecum samples of colitis non-induced Gankyrin ${ }^{\text {fff }}$ control mice (FDR < 0.05). Each dot represents the relative abundance of OTUs within each sample.

Additional file 5: Figure S5. Bacterial OTUs under-represented in rectum samples of colitis non-induced Villin-Cre;Gankyrin ${ }^{f / f}$ mice in comparison with rectum samples of colitis non-induced Gankyrin ${ }^{\mathrm{f} / \mathrm{f}}$ control mice 
(FDR < 0.05). Each dot represents the relative abundance of OTUs within each sample.

Additional file 6: Figure S6. Bacterial OTUs over-represented in rectum samples of colitis non-induced Villin-Cre;Gankyrin ${ }^{f / f}$ mice in comparison with rectum samples of colitis non-induced Gankyrin ${ }^{\mathrm{f} / f}$ control mice (FDR $<0.05)$. Each dot represents the relative abundance of OTUs within each sample.

Additional file 7: Figure S7. Principal coordinate analysis of (A) unweighted UniFrac distance and (B) weighted UniFrac distance between all samples, including samples from cohouse mice, based on bacterial operational taxonomic units (OTUs). The color codes for the samples are, light blue: co-housed colitis-induced Gankyrin ${ }^{f / f}$ control mice, pink: cohoused colitis-induced Villin-Cre;Gankyrin ${ }^{\text {f/f }}$ mice, green: colitis noninduced Gankyrin ${ }^{\mathrm{f} / \mathrm{f}}$ control mice, orange: colitis non-induced VillinCre;Gankyrin ${ }^{f / f}$ mice, blue: colitis-induced Gankyrin ${ }^{f / f}$ control mice, red: colitis-induced Villin-Cre;Gankyrin ${ }^{\text {fff }}$ mice.

Additional file 8: Figure S8. Bacterial OTUs showing differential abundance in comparison between the rectum samples of co-housed colitis-induced Villin-Cre;Gankyrin f/f mice vs. co-housed colitis-induced Gankyrin $^{f / f}$ control mice (FDR < 0.05). Each dot represents the relative abundance of OTUs within each sample.

Additional file 9: Table S1. The corrected $P$-values and effect sizes reported by the Adonis test when performed on weighted unifrac distances between sample groups

Additional file 10: Table S2. Number of sequence reads analyzed during the $16 \mathrm{~S}$ rRNA amplicon analysis conducted to understand the intestinal microbiota in the co-housed mice groups

Additional file 11: Table S3. Richness and Shannon diversity estimates of intestinal microbiota of co-housed Gankyrin ${ }^{f / f}$ control mice and cohoused colitis induced Villin-Cre;Gankyrin ${ }^{f / f}$ mice

\section{Acknowledgements}

Computational time was provided by the SuperComputer System, Institute for Chemical Research, Kyoto University.

\section{Authors' contributions}

T.S. conceived the original idea, carried out the experiment and wrote the manuscript. H.N. conducted bioinformatics analysis of 165 rRNA amplicon sequences and interpreted the results; contributed to the writing and generation of the figures; rendered intellectual inputs on the work. T.N. carried out the experiment and discussed the results. S.G. supervised bioinformatics analysis; contributed to the writing. H.O. supervised bioinformatics analysis and interpreted the results; contributed to the writing; rendered intellectual inputs on the work. M.K. discussed the results and supervised the project. All authors have read and approved the manuscript

\section{Funding}

Animal experiments were supported by grants from the Japanese Foundation for Research and Promotion of Endoscopy (JFE) and microbiota experiments were supported by grants from JSPS KAKENHI Grant Number (JP26460979, 17 K09396, 17 H06404 and 16KT0020) and Grants-in-Aid for Scientific Research on Innovative Area "Frontier Research on Chemical Communications (FY2017-2021). H.N. was supported by Grant-in-Aid for JSPS Research Fellow (19 J14228).

\section{Availability of data and materials}

Sequence data of the raw paired-end reads were deposited to DNA Data Bank of Japan/Sequence Read Archive (DDBJ/DRA) and are accessible through the accession number DRA009279. The merged reads can be obtained from the following site: ftp://ftp.genome.jp/pub/db/community/ microbiome_kindai/.

\section{Ethics approval and consent to participate}

The human study (endoscopies and samples taken) and the animal study protocols were approved by the Medical Ethics Committee of Kindai University Faculty of Medicine and Institutional Animal Care (\#23-098, \#28-
224). Written informed consent for the human study (endoscopies and samples taken) was obtained.

\section{Consent for publication}

Not applicable.

\section{Competing interests}

The authors declare no conflict of interest.

\section{Author details}

'Department of Gastroenterology and Hepatology, Kindai University Faculty of Medicine, 377-2, Osaka-Sayama, Osaka 589-8511, Japan. ${ }^{2}$ Institute for Chemical Research, Kyoto University, Uji 611-0011, Japan. ${ }^{3}$ Database Center for Life Science, Joint-Support Center for Data Science Research, Research Organization of Information and Systems, Wakashiba, Kashiwa, Chiba 277-0871, Japan.

Received: 19 February 2019 Accepted: 29 December 2019 Published online: 15 January 2020

\section{References}

1. Baumgart DC, Sandborn WJ. Inflammatory bowel disease: clinical aspects and established and evolving therapies. Lancet. 2007;369:1641-57.

2. Lopetuso LR, Petito V, Graziani C, Schiavoni E, Paroni Sterbini F, Poscia A, et al. Gut Microbiota in Health, Diverticular Disease, Irritable Bowel Syndrome, and Inflammatory Bowel Diseases: Time for Microbial Marker of Gastrointestinal Disorders. Dig Dis. 2018;36:56-65.

3. Itzkowitz SH, Yio X. Inflammation and Cancer IV. Colorectal cancer in inflammatory bowel disease: the role of inflammation. Am J Physiol Gastrointest Liver Physiol. 2004;287:7-17.

4. Ullman TA, Itzkowitz SH. Intestinal Inflammation and Cancer. Gastroenterology. 2011;140:1807-16.

5. Sakurai T, Kashida H, Watanabe T, Hagiwara S, Mizushima T, lijima H. Stress Response Protein Cirp Links Inflammation and Tumorigenesis in ColitisAssociated Cancer. Cancer Res. 2014;74:6119-28.

6. Adachi T, Sakurai T, Kashida H, Mine H, Hagiwara S, Matsui S, et al. Involvement of Heat Shock Protein A4/Apg-2 in Refractory Inflammatory Bowel Disease. Inflamm Bowel Dis. 2015;21:31-9.

7. Sakurai T, Kashida H, Komeda Y, Nagai T, Hagiwara S, Watanabe T, et al. Stress Response Protein RBM3 Promotes the Development of Colitisassociated Cancer. Inflamm Bowel Dis. 2017;23:66-74.

8. Sakurai T, Higashitsuji H, Kashida H, Watanabe T, Komeda $Y$, Nagai T, et al. The oncoprotein gankyrin promotes the development of colitis-associated cancer through activation of STAT3. Oncotarget. 2017;8:24762-76.

9. Yamada M, Sakurai T, Komeda Y, Nagai T, Kamata K, Minaga K, et al. Clinical significance of Bmi1 expression in inflammatory bowel disease. Oncology. 2017:93:20-6.

10. Sakurai T, Yada N, Hagiwara S, Arizumi T, Minaga K, Kamata K, et al. Gankyrin induces STAT3 activation in tumor microenvironment and sorafenib resistance in hepatocellular carcinoma. Cancer Sci. 2017;108:1996-2003.

11. Sakurai T, Kudo M, Umemura A, He G, Elsharkawy AM, Seki E, et al. p38a Inhibits Liver Fibrogenesis and Consequent Hepatocarcinogenesis by Curtailing Accumulation of Reactive Oxygen Species. Cancer Res. 2013;73:215-24.

12. Park AM, Kudo M, Hagiwara S, Tabuchi M, Watanabe T, Munakata H, et al. p38MAPK suppresses chronic pancreatitis by regulating HSP27 and BAD expression. Free Radic Biol Med. 2012;52:2284-91.

13. Illumina Inc. 165 metagenomic sequencing library preparation. Illumina Inc 2013. http://jp.support.illumina.com/downloads/16s_metagenomic_ sequencing_library_preparation.html. Accessed 15 July 2015.

14. Nishiyama $H$, Nagai $T$, Kudo M, Okazaki $Y$, Azuma $Y$, Watanabe $T$, et al. Supplementation of pancreatic digestive enzymes alters the composition of intestinal microbiota in mice. Biochem Biophys Res Commun. 2018;495:273-9.

15. Bolger AM, Lohse M, Usadel B. Trimmomatic: a flexible trimmer for Illumina sequence data. Bioinformatics. 2014;30:2114-20.

16. Martin M. Cutadapt Removes Adapter Sequences From High-Throughput Sequencing Reads. EMBnet.J. 2011;17:10-2.

17. Magoč T, Salzberg SL. FLASH: fast length adjustment of short reads to improve genome assemblies. Bioinformatics. 2011;27:2957-63. 
18. McDonald D, Price MN, Goodrich J, Nawrocki EP, DeSantis TZ, Probst A, et al. An improved Greengenes taxonomy with explicit ranks for ecologica and evolutionary analyses of bacteria and archaea. ISME J. 2012;6:610-8.

19. Edgar RC. Search and clustering orders of magnitude faster than BLAST. Bioinformatics. 2010;26:2460-1.

20. Caporaso JG, Kuczynski J, Stombaugh J, Bittinger K, Bushman FD, Costello EK, et al. QIIME allows analysis of high-throughput community sequencing data. Nat Methods. 2010;7:335-6.

21. Edgar RC, Haas BJ, Clemente JC, Quince C, Knight R. UCHIME improves sensitivity and speed of chimera detection. Bioinformatics. 2011;27:2194200.

22. Rognes T, Flouri T, Nichols B, Quince C, Mahé F. VSEARCH: a versatile open source tool for metagenomics. PeerJ. 2016. https://doi.org/10.7717/peerj. 2584.

23. Wang Q, Garrity GM, Tiedje JM, Cole JR. Naive Bayesian classifier for rapid assignment of rRNA sequences into the new bacterial taxonomy. Appl Environ Microbiol. 2007:73:5261-7.

24. Shannon CE. A mathematical theory of communication. Bell Syst Tech J. 1948;27:379-423.

25. Lozupone C, Knight R. UniFrac: a new phylogenetic method for comparing microbial communities. Appl Environ Microbiol. 2005;71:8228-35.

26. Nawrocki EP, Kolbe DL, Eddy SR. Infernal 1.0: inference of RNA alignments. Bioinformatics. 2009;25:1335-7.

27. Price MN, Dehal PS, Arkin AP. FastTree 2-approximately maximum-likelihood trees for large alignments. PLoS One. 2010. https://doi.org/10.1371/journal. pone.0009490

28. Anderson MJ. A new method for non-parametric multivariate analysis of variance. Austral Ecol. 2001;26:32-46.

29. Love Ml, Huber W, Anders S. Moderated estimation of fold change and dispersion for RNA-seq data with DESeq2. Genome Biol. 2014;15:550.

30. Zhang Z, Schwartz S, Wagner L, Miller W. A greedy algorithm for aligning DNA sequences. J Comput Biol. 2000;7:203-14.

31. Johnson M, Zaretskaya I, Raytselis Y, Merezhuk Y, McGinnis S, Madden TL. NCBI BLAST: a better web interface. 2008. https://doi.org/10.1093/nar/ gkn201.

32. Maynard CL, Elson CO, Hatton RD, Weaver CT. Reciprocal interactions of the intestinal microbiota and immune system. Nature. 2012;489:231-41.

33. Mine H, Sakurai T, Kashida H, Matsui S, Nishida N, Nagai T, et al. Association of Gankyrin and Stemness Factor Expression in Human Colorectal Cancer. Dig Dis Sci. 2013;58:2337-44.

34. Qian YW, Chen Y, Yang W, Fu J, Cao J, Ren YB, et al. p28 ${ }^{\text {GANK }}$ Prevents Degradation of Oct4 and Promotes Expansion of Tumor-Initiating Cells in Hepatocarcinogenesis. Gastroenterology. 2012;142:1547-58.e14.

35. Ostaff MJ, Stange EF, Wehkamp J. Antimicrobial peptides and gut microbiota in homeostasis and pathology. EMBO Mol Med. 2013;5:1465-83.

36. Kostic AD, Xavier RJ, Gevers D. The microbiome in inflammatory bowel disease: current status and the future ahead. Gastroenterology. 2014;146: 1489-99.

37. Maeda S, Kamata H, Luo JL, Leffert H, Karin M. IKKbeta Couples Hepatocyte Death to Cytokine-Driven Compensatory Proliferation that Promotes Chemical Hepatocarcinogenesis. Cell. 2005:121:977-90.

38. Greten FR, Eckmann L, Greten TF, Park JM, Li ZW, Egan LJ, et al. IKKbeta Links Inflammation and Tumorigenesis in a Mouse Model of ColitisAssociated Cancer. Cell. 2004;118:285-96.

39. Sakurai T, He G, Matsuzawa A, Yu GY, Maeda S, Hardiman G, et al. Hepatocyte Necrosis Induced by Oxidative Stress and IL-1 alpha Release Mediate Carcinogen-Induced Compensatory Proliferation and Liver Tumorigenesis. Cancer Cell. 2008;14:156-65.

40. Shen Z, Feng Y, Muthupalani S, Sheh A, Cheaney LE, Kaufman CA. Novel Helicobacter species H.japonicum isolated from laboratory mice from Japan induces typhlocolitis and lower bowel carcinoma in C57BL/129 IL10-/mice. Carcinogenesis. 2016;37:1190-8.

41. Yu Q, Zhang S, Li L, Xiong L, Chao K, Zhong B, et al. Enterohepatic Helicobacter species as a potential causative factor in inflammatory bowel disease: a meta-analysis. Medicine. 2015;94:e1773.

42. Rokkas T, Gisbert JP, Niv Y, O'Morain C. The association between Helicobacter pylori infection and inflammatory bowel disease based on meta-analysis. United European Gastroenterol J. 2015;3:539-50.

43. Warren YA, Citron DM, Merriam CV, Goldstein EJ. Biochemical differentiation and comparison of Desulfovibrio species and other phenotypically similar genera. J Clin Microbiol. 2005;43:4041-5.
44. Singh SB, Lin HC. Hydrogen sulfide in physiology and diseases of the digestive tract. Microorganisms. 2015:3:866-89.

45. Beaumont M, Andriamihaja M, Lan A, Khodorova N, Audebert M, Blouin JM, et al. Detrimental effects for colonocytes of an increased exposure to luminal hydrogen sulfide: the adaptive response. Free Radic Biol Med. 2016; 93:155-64.

46. Salzman NH. Paneth cell defensins and the regulation of the microbiome: détente at mucosal surfaces. Gut Microbes. 2010;1:401-6.

\section{Publisher's Note}

Springer Nature remains neutral with regard to jurisdictional claims in published maps and institutional affiliations.
Ready to submit your research? Choose BMC and benefit from:

- fast, convenient online submission

- thorough peer review by experienced researchers in your field

- rapid publication on acceptance

- support for research data, including large and complex data types

- gold Open Access which fosters wider collaboration and increased citations

- maximum visibility for your research: over $100 \mathrm{M}$ website views per year

At BMC, research is always in progress.

Learn more biomedcentral.com/submissions 